\title{
Status of ATLAS
}

Peter Jenni ${ }^{1}$

CERN

CH-1211 Geneva 23, Switzerland

peter.jenni@cern.ch

\section{On behalf of the ATLAS Collaboration}

The general status of the ATLAS experiment at the LHC is briefly reviewed in this report. An account is given on the main milestones of the installation of the detector, as well as of the preparation for the data collection and analysis framework in the worldwide Collaboration. First results from the in-situ commissioning of the detector, and details of the physics potential are reported in more than 20 dedicated talks during this 'Physics at LHC 2008' conference.

2008 Physics at LHC

Split, Croatia

September 29-4 October 2008

\footnotetext{
1 Peter Jenni, CERN, Spokesperson of the ATLAS Collaboration
} 


\section{The ATLAS Collaboration}

ATLAS is a worldwide Collaboration, spanning over the whole globe. As of July 2008 there are about 2500 scientific authors working on the project, including some 700 students. The constituencies of the ATLAS Collaboration are 169 Institutions from 37 countries, where Institutions in some cases group together several universities of a given country. All ATLAS Collaboration Institutions are listed below.

Albany, Alberta, NIKHEF Amsterdam, Ankara, LAPP Annecy, Argonne NL, Arizona, UT Arlington, Athens, NTU Athens, Baku, IFAE Barcelona, Belgrade, Bergen, Berkeley LBL and UC, HU Berlin, Bern, Birmingham, UAN Bogota, Bologna, Bonn, Boston, Brandeis, Bratislava/SAS Kosice, Brookhaven NL, Buenos Aires, Bucharest, Cambridge, Carleton, CERN, Chinese Cluster, Chicago, Chile, Clermont-Ferrand, Columbia, NBI Copenhagen, Cosenza, AGH UST Cracow, IFJ PAN Cracow, UT Dallas, DESY, Dortmund, TU Dresden, JINR Dubna, Duke, Frascati, Freiburg, Geneva, Genoa, Giessen, Glasgow, Göttingen, LPSC Grenoble, Technion Haifa, Hampton, Harvard, Heidelberg, Hiroshima, Hiroshima IT, Indiana, Innsbruck, Iowa SU, Irvine UC, Istanbul Bogazici, KEK, Kobe, Kyoto, Kyoto UE, Lancaster, UN La Plata, Lecce, Lisbon LIP, Liverpool, Ljubljana, QMW London, RHBNC London, UC London, Lund, UA Madrid, Mainz, Manchester, CPPM Marseille, Massachusetts, MIT, Melbourne, Michigan, Michigan SU, Milano, Minsk NAS, Minsk NCPHEP, Montreal, McGill Montreal, RUPHE Morocco, FIAN Moscow, ITEP Moscow, MEPhI Moscow, MSU Moscow, Munich LMU, MPI Munich, Nagasaki IAS, Nagoya, Naples, New Mexico, New York, Nijmegen, BINP Novosibirsk, Ohio SU, Okayama, Oklahoma, Oklahoma SU, Olomouc, Oregon, LAL Orsay, Osaka, Oslo, Oxford, Paris VI and VII, Pavia, Pennsylvania, Pisa, Pittsburgh, CAS Prague, CU Prague, TU Prague, IHEP Protvino, Regina, Ritsumeikan, UFRJ Rio de Janeiro, Rome I, Rome II, Rome III, Rutherford Appleton Laboratory, DAPNIA Saclay, Santa Cruz UC, Sheffield, Shinshu, Siegen, Simon Fraser Burnaby, SLAC, Southern Methodist Dallas, NPI Petersburg, Stockholm, KTH Stockholm, Stony Brook, Sydney, AS Taipei, Tbilisi, Tel Aviv, Thessaloniki, Tokyo ICEPP, Tokyo MU, Toronto, TRIUMF, Tsukuba, Tufts, Udine/ICTP, Uppsala, Urbana UI, Valencia, UBC Vancouver, Victoria, Washington, Weizmann Rehovot, FH Wiener Neustadt, Wisconsin, Wuppertal, Würzburg, Yale, Yerevan.

\section{The main components of the ATLAS detector}

The overall ATLAS detector layout is shown in Figure 1. A very comprehensive description, including considerable technical details, of the ATLAS detector is provided in Ref. 1. This discusses the detector 'as built and installed in the underground cavern' at Point-1 of the LHC. It also demonstrates its performance based on many years of test beam measurements with real detector components.

The ATLAS detector is forward-backward symmetric with respect to the interaction

point. The magnet configuration comprises a thin superconducting solenoid surrounding the inner-detector cavity, and three large superconducting toroids (one barrel and two end-caps) 


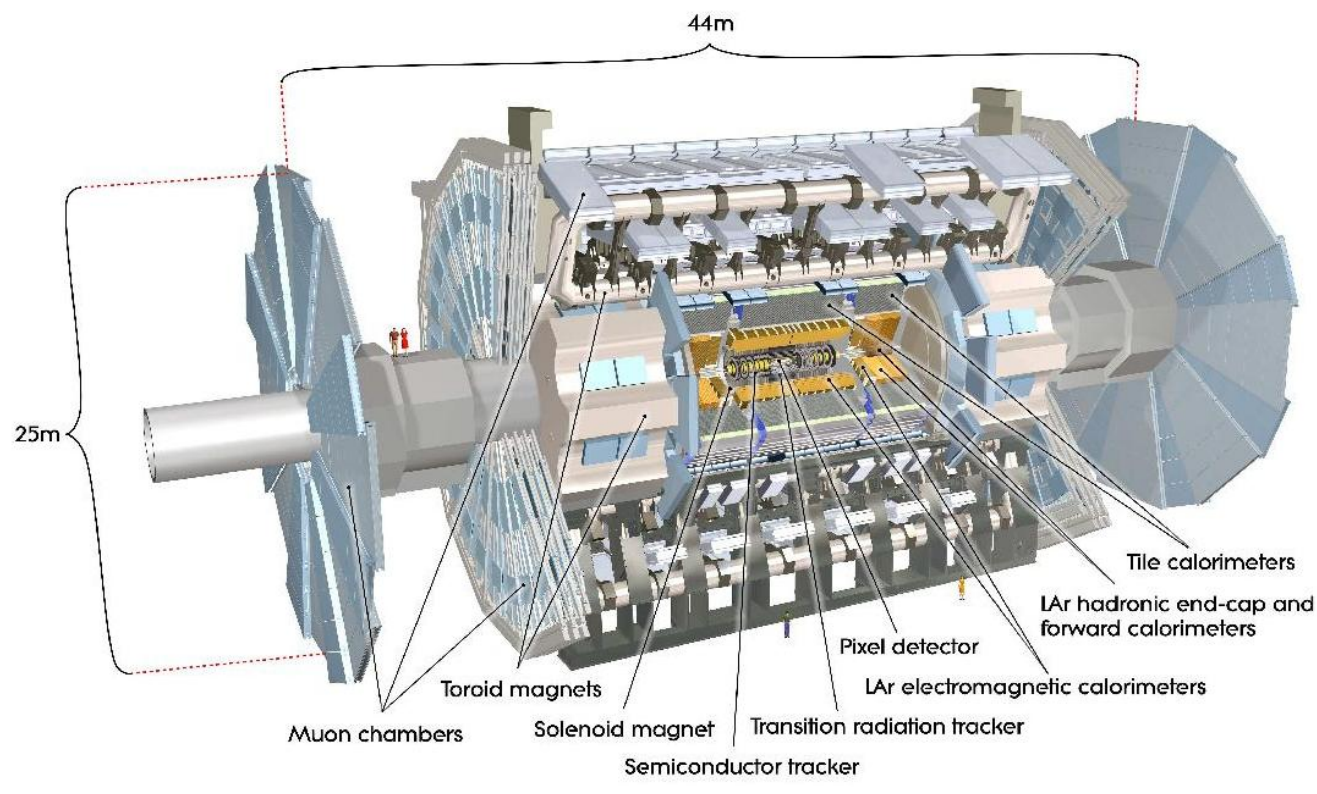

Figure 1: Layout of the ATLAS detector.

arranged with an eight-fold azimuthal symmetry around the calorimeters. This fundamental choice has driven the design of the rest of the detector.

The inner detector is immersed in a $2 \mathrm{~T}$ solenoidal field. Pattern recognition, momentum and vertex measurements, and electron identification are achieved with a combination of discrete, high-resolution semiconductor pixel and strip detectors in the inner part of the tracking volume, and straw-tube tracking detectors with the capability to generate and detect transition radiation in its outer part.

High granularity liquid-argon (LAr) electromagnetic sampling calorimeters, with excellent performance in terms of energy and position resolution, cover the pseudorapidity range $|\eta|<3.2$. The hadronic calorimetry in the range $|\eta|<1.7$ is provided by a scintillator-tile calorimeter, which is separated into a large barrel and two smaller extended barrel cylinders, one on either side of the central barrel. In the end-caps $(|\eta|>1.5)$, LAr technology is also used for the hadronic calorimeters, matching the outer $|\eta|$ limits of end-cap electromagnetic calorimeters. The LAr forward calorimeters provide both electromagnetic and hadronic energy measurements, and extend the pseudorapidity coverage to $|\eta|=4.9$.

The calorimeter is surrounded by the muon spectrometer. The air-core toroid system, with a long barrel and two inserted end-cap magnets, generates strong bending power in a large volume within a light and open structure. Multiple-scattering effects are thereby minimised, and excellent muon momentum resolution is achieved with three layers of high precision tracking chambers. The muon instrumentation includes trigger chambers with timing resolution of the order of 1.5-4 ns. The muon spectrometer defines the overall dimensions of the ATLAS detector.

A series of detectors cover the forward regions on both sides of the ATLAS detector near the beam line, with the aim of measuring and monitoring the LHC luminosity, as well as 
providing physics measurements in the very forward regions. For the start-up they are only partially installed: at $17 \mathrm{~m}$ from the interaction point there are luminosity monitor detectors based on Cherenkov tubes (LUCID), at $140 \mathrm{~m}$ is a zero degree calorimeter for detecting photons and neutrons mainly in Heavy Ion collisions, and at $240 \mathrm{~m}$ precision tracking detectors in Roman Pots will measure elastic scattering at very small angles for a total cross-section determination.

The proton-proton interaction rate at the design luminosity of $10^{34} \mathrm{~cm}^{-2} \mathrm{~s}^{-1}$ is approximately $1 \mathrm{GHz}$, while the event data recording, based on technology and resource limitations, is limited to about $200 \mathrm{~Hz}$. This requires an overall rejection factor of $5 \times 10^{6}$ against minimum-bias processes while maintaining maximum efficiency for the new physics. The Level-1 (L1) trigger system uses a subset of the total detector information to make a decision on whether or not to continue processing an event, reducing the data rate to approximately $75 \mathrm{kHz}$ (limited by the bandwidth of the readout system, which is upgradeable to $100 \mathrm{kHz}$ ). The subsequent two levels, collectively known as the high-level trigger (HLT), are the Level-2 (L2) trigger and the event filter. They provide the reduction to a final data-taking rate of approximately $200 \mathrm{~Hz}$.

\section{Installation and commissioning of the detector components}

The excavation and completion of the underground cavern took five years, from 1998 to 2003. In summer 2003 began the installation of the detector components proper, spanning over another five-year period with intense and complex underground activities. The components were lowered into the cavern, with its floor about $90 \mathrm{~m}$ underground, through two shafts at either end of the hall and then assembled into their final position in the ATLAS detector.

The barrel toroid was the first to be completed with the last coil put in place in August 2005. In parallel, the preassembled LAr calorimeters in their cryostats were lowered into the cavern and the tile calorimeter modules were mounted around them to form the barrels. By May 2006, the central and both extended barrels were completed and had been moved to their location inside the toroid (see Figure 2, left). At the same time, the barrel toroid structure and the big end-cap wheels were progressively equipped with muon chambers (see Figure 2, right). The small muon end-cap wheels were mounted on surface and lowered through the shafts, as well as the toroid end-cap cryostats. The process culminated with the installation of the last muon chamber in July 2008. The inner detector was installed in various phases: TRT and SCT barrel in August 2006, end-caps in May 2007 and the Pixel detector in June 2007 (see Figure 3). A very major challenge consisted in the installation of more than 50'000 cables with more than 3'000 km length, and more than 10'000 pipes for services. Historical moments were passed on 16th June 2008 with the closure of the LHC beam pipe and then early August 2008 with the successful bake-out of the beam pipe. The latter was particularly critical because it required the evaporative cooling system fully operational to protect the Pixel layers from overheating. The evaporative cooling plant suffered a major failure of its compressors at the beginning of May 2008, and the repair and cleaning of the plant was dictating very much the critical path for the closure of the detector. 
After installation the detector components were connected to their readout electronics, operated with their services (cooling, gas) and integrated gradually into the global Trigger and Data Acquisition system. Over the last years they were brought into operation as soon as installed and connected. In all cases this in-situ commissioning was preceded by very extensive tests and adjustments at the surface, and in test beams.
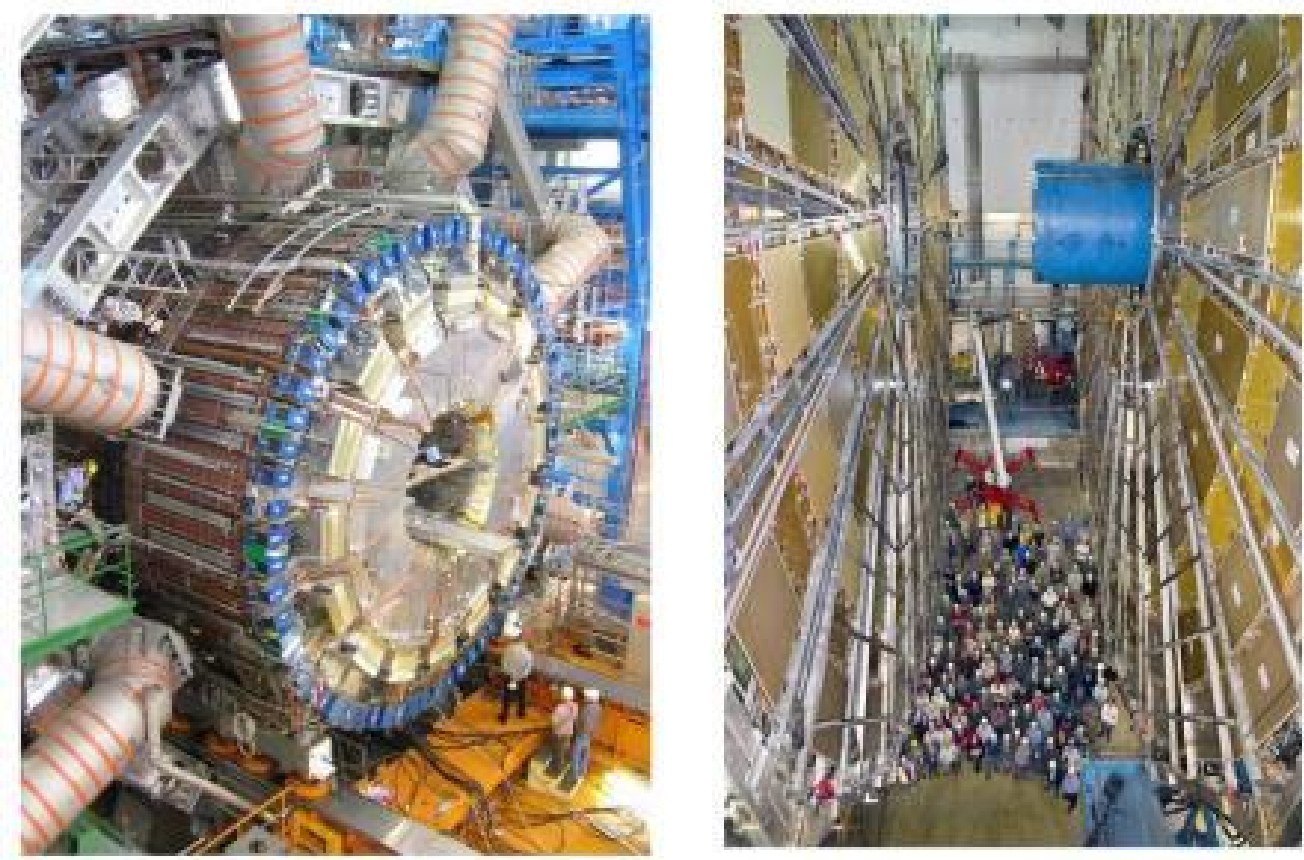

Figure 2: Left: the barrel calorimeter being moved into position inside the toroid in November 2005. Right: the large end-cap muon wheels after completion of the installation in September 2007.
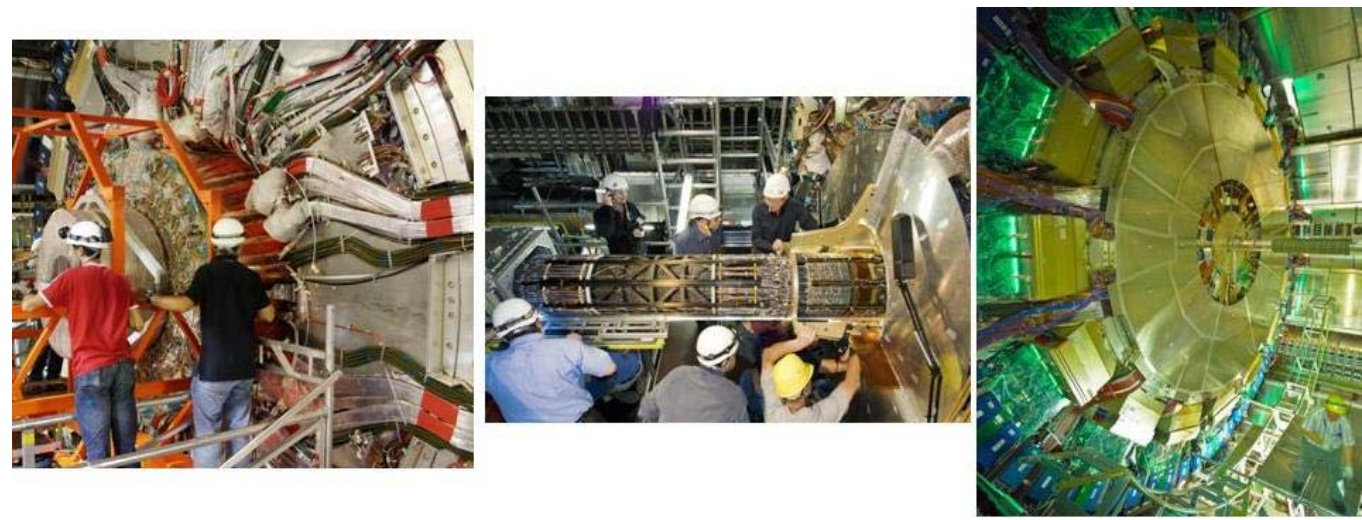

Figure 3: Left: the barrel TRT and SCT being installed in August 2006. Middle: the Pixel detector being inserted in June 2007. Right: ID volume sealed with the end-plate in April 2008. 
The first fully tested component of the Magnet System was the central solenoid which was installed as an integrated part of the barrel LAr calorimeter. In summer 2006 it was operated at full current $(7.7 \mathrm{kA})$, and a field mapping was done with a precision in $\Delta \mathrm{B} / \mathrm{B}$ close to $10^{-4}$. All the eight single coils of the barrel toroid were fully tested on the surface, as well as partially the two end-cap toroids. A complete system test campaign of all magnets started in summer 2008 and was successfully concluded in August 2008, after some training quenches in the end-cap toroid coils. Since then the toroid system was operated routinely at $20.4 \mathrm{kA}$.

For the LHC start-up in September 2008 the detector was operational to a very large extent. Because of the cooling failure during several months earlier in the year, mentioned above, the Pixel and SCT sub-systems had only very limited in-situ commissioning time available. Nevertheless, more than $95 \%$ of the Pixel modules, $99.8 \%$ of the barrel, and $97.6 \%$ of the end-cap SCT modules were operational. The TRT was read out at the $98 \%$ level. The calorimeter commissioning was already in a mature state given the up to two years of operational experience since underground installation. The LAr calorimeters had only $0.02 \%$ of isolated dead channels, plus some $0.8 \%$ of dead read-out channels, including one missing (out of eight) hadronic end-cap low voltage power supplies which failed after closure of the detector. The tile calorimeter operated with $0.2 \%$ isolated dead cells and 2 out of 256 sectors off due to power supply problems. Finally the muon spectrometer instrumentation had less than $1 \%$ problematic precision chambers, with more than $99 \%$ of the alignment lines working. For the trigger chambers all TGC were working, and three of the 16 RPC sectors were still under final timing adjustments.

The full chain of the Trigger and DAQ chain was operational, albeit with only $35 \%$ of the HLT processing power installed (following the staging plans for the experiment).

\section{Computing and distributed analysis}

The Collaboration-wide distributed computing infrastructure is fully embedded into the framework of the worldwide LHC Computing Grid (wLCG) of which ATLAS is a very active partner. However, it must be noted that in addition to this Grid infrastructure there is a very sizable experiment-specific effort required to efficiently interface the ATLAS software suite and analysis framework to the wLCG infrastructure.

During the past years ATLAS and wLCG successfully performed large data transfer exercises, from the Tier-0 to all Tier-1s and Tier-2s. During the first half of 2008 the most significant such exercises were the Common Computing Readiness Challenge (CCRC) phase-2 run at the end of May 2008 together with the other LHC experiments, and the second phase of the ATLAS-internal Full Dress Rehearsal with large simulated data samples which 'stresstested' the full data flow, various calibration and data quality steps, and distributed analysis using the whole Tier-1, Tier-2 and Tier-3 structures. The whole computing and software chain has been operational since the continuous cosmic ray data taking which started in summer 2008, and it demonstrated its efficiency during the first LHC beam induced data taken on the LHC start-up 10th-11th September 2008. 


\section{Cosmic ray data, and first 'LHC beam-splash’ events recorded}

The ATLAS detector was operational and ready to meet the LHC start-up. The full detector had been operated for a few months collecting cosmic ray data before the first single beam injection into the LHC. The experiment was kept operational continuously since end of August 2008, typically recording cosmic ray data during nights and weekends, complemented with dedicated sub-system commissioning work during week-day periods.

As a highlight, the first beam-related events were successfully recorded and reconstructed on 10th September 2008 during the official LHC start-up day. These first LHC beam-splash events, as well as the few beam-halo background events from the first stored single LHC beam runs, were used very efficiently for initial timing adjustments. Figure 4 (left) displays such a beam-halo event.

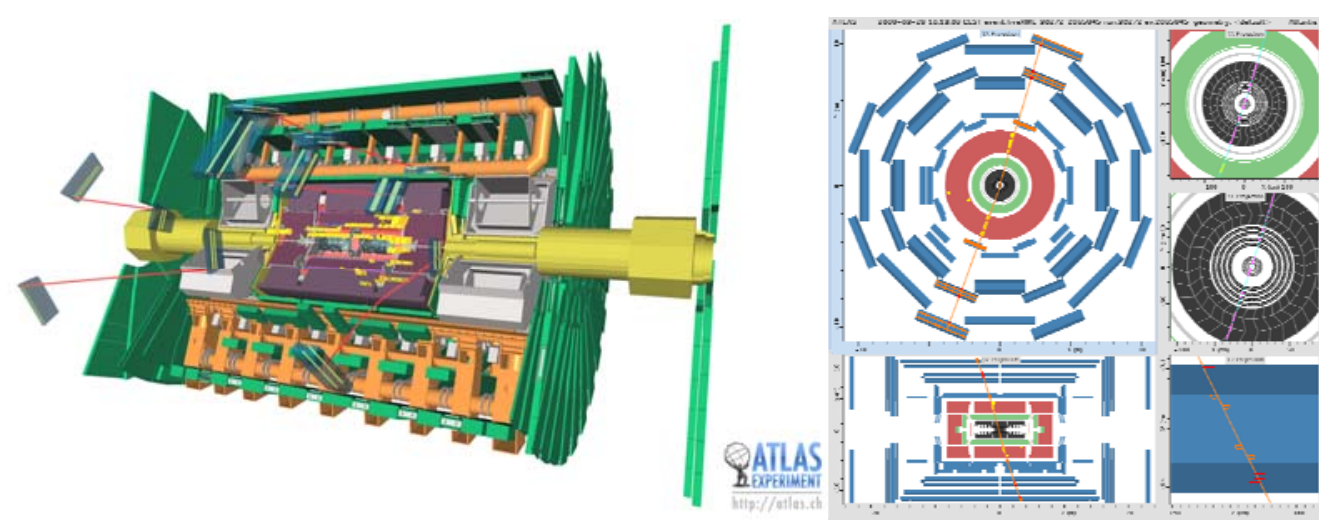

Figure 4: Left: beam-halo event from single LHC beam operation on 10th September 2008. Right: cosmic ray muon traversing the full ATLAS detector.

Since the LHC incident, which occurred on $19^{\text {th }}$ September 2008, the full detector was essentially continuously operated in cosmic ray data collection mode. Two hundred million cosmic ray triggers (Figure 4, right) have been collected with the full detector, with about 100k muons also passing through the smallest-volume detector, the Pixels. These events are very valuable for improving monitoring and data quality procedures, as well as for initial global alignments and calibrations, as will be described in several dedicated talks at this conference.

The plan is to open the detector starting in December 2008 to access the detector components which require interventions, in order to repair as much as possible the small fraction of problematic channels mentioned in Section 3. The detector will then be closed again in May 2009 for resuming operation with LHC beams.

\section{Reference}

[1] ATLAS Collaboration, G. Aad et al., The ATLAS Experiment at the CERN Large Hadron Collider, JINST 3 (2008) S08003. 